\title{
The size of the stability regions of Jupiter Trojans
}

\author{
F. Freistetter
}

\author{
Astrophysikalisches Institut, Universität Jena, Schillergässchen 2-3, 07745 Jena, Germany \\ e-mail: florian@astro.uni-jena.de
}

Received 14 December 2005 / Accepted 15 February 2006

\section{ABSTRACT}

\begin{abstract}
Context. We have known about the Trojan group of asteroids since 1906. With a suspected number comparable to that of the mainbelt, they move around the Lagrangian points $L_{4}$ and $L_{5}$ of the Sun-Jupiter system.

Aims. During the past century the Trojan asteroids were the topic of many studies of their orbital dynamics and stability. In recent years it has been possible to perform extensive numerical simulations of the motion of these asteroids and thus also to investigate the size and structure of the stability region around the Lagrangian points. The aim of this work is to find new numerical estimates of the size and shape of the stability region. Additionally, the problem of the asymmetry between the two Trojan groups (there seem to be more asteroids in $L_{4}$ than in $L_{5}$ ) is investigated.

Methods. We investigate the extension of this regions in the $(\sigma-e)$ and $(\sigma-i)$ plane (where $\left.\sigma=(\omega+\Omega+M)-\left(\omega_{\mathrm{JUP}}+\Omega_{\mathrm{JUP}}+M_{\mathrm{JUP}}\right)\right)$ and show how the initial inclination and initial eccentricity change the size and shape of this region. The problem of Trojan asymmetry is investigated by directly analyzing the evolution of orbital parameters.

Results. We find both that there is a "critical" eccentricity of $e_{\mathrm{INI}} \approx 0.15$ that plays an important role in the dynamics of Trojan asteroids and influences the structure of the stability regions and that the region of the small amplitudes of $\Delta \sigma=\sigma_{\max }-\sigma_{\min }$ is larger for $L_{4}$ due to the influence of Saturn and thus the $L_{4}$ group of Trojans seems to be more stable than the $L_{5}$ group.
\end{abstract}

Key words. celestial mechanics - minor planets, asteroids - methods: $N$-body simulations

\section{Introduction}

Since the first discovery of a Trojan asteroid by Max Wolf in Heidelberg in 1906, the problem of the motion around the Lagrangian point evolved from what was a purely theoretical case study into an important part of Solar System dynamics. One of the first studies of the stability regions around the Lagrangian points was made by Rabe $(1967,1968)$. Érdi then investigated the motion of Trojans analytically in many papers (e.g. Érdi 1981, 1984, 1988, 1996, 1997).

Other analytical studies were done by Bien (1980) for Trojans with high inclinations, by Bien \& Schubart (1984) for the influence of secular resonances, by Schubart \& Bien (1987) for the relation between the orbital elements, and by Namouni (1999), Namouni et al. (1999), and Namouni \& Murray (2000), who investigated the effect of eccentricity and inclination on the motion of Trojans. Numerical integrations to investigate their motion of were performed e.g. by Mikkola \& Innanen (1992, 1995) and Marzari et al. (2003). Morais (1999) derived a secular theory for the Trojan type motion, Giorgilli \& Skokos (1997) used the Nekhoroshev theorem to investigate the stability regions, and several authors used semi-analytical or mapping methods to study the dynamics of Trojans (e.g. Celletti \& Giorgilli 1991; Skokos \& Dokoumetzidis 2001; Beaugé \& Roig 2001; Sándor et al. 2002; Sándor \& Érdi 2003; Efthymiopoulos 2005). Milani $(1993,1994)$ used numerical methods to calculate the proper elements of the Trojans and showed that the orbits of some real ones are chaotic (the chaoticity of some Trojan orbits have also been mentioned earlier by Shoemaker et al. 1989). These chaotic orbits and the connected escape of Trojan asteroids were also studied numerically by many authors (e.g. Pilat-Lohinger et al. 1999; Dvorak \& Tsiganis 2000; Tsiganis et al. 2000; Marzari \& Scholl 2002).

Due to the increasing number of discovered Trojans and the increase in computer power, it is now possible to numerically investigate the stability regions around the Lagrangian points in great detail and extend the early analytical borders obtained by Rabe (1967). Such studies were performed e.g. by Levison et al. (1997), Schwarz \& Gyergyovits (2003), Brasser et al. (2004), Schwarz et al. (2004), Dvorak \& Schwarz (2005), Tsiganis et al. (2005), and Robutel et al. (2005). An additional problem that is closely connected to these studies is the different number of Trojans observed near $L_{4}$ and $L_{5}$. There seem to be significantly more near $L_{4}$ than $L_{5}{ }^{1}$, the reason for this behavior is still unknown (see Sect. 5 for details).

The present work has two main goals. First, we want to extend the existing studies of the stability regions around the Lagrangian points and, second, we want to search for real numerical evidence of a dynamical difference between $L_{4}$ and $L_{5}$ Trojans that can be seen directly in the evolution of the orbital elements. Thus, Sect. 2 will give an overview on the methods and models used in this work; Sect. 3 will investigate the motion in the direct vicinity of the Lagrangian points; Sect. 4 will investigate the structure of the stability regions, and Sect. 5 will review the problem of the different number of $L_{4}$ and $L_{5}$ Trojans and use the results obtained during this work to get some new insights.

\section{Methods}

The dynamical evolution of many Trojan asteroids shows chaotic features; see e.g. Milani (1993), Milani et al. (1997) for an

\footnotetext{
${ }^{1}$ As of March 6, there are $1121 L_{4}$ Trojans and $885 L_{5}$ Trojans.
} 
Table 1. Models and initial conditions.

\begin{tabular}{|c|c|c|c|c|c|c|}
\hline Model & eccentricity $e$ & inclination $i$ & libration $\sigma$ & $\overline{T_{\text {int }}}$ & Perturbers & Method \\
\hline Model 1 & {$[0: 0.2] \delta e=0.01$} & {$\left[0^{\circ}: 30^{\circ}\right] \delta i=1^{\circ}$} & $60^{\circ}, 300^{\circ}$ & $10 \mathrm{Myr}$ & OSS & B-S, HYB, LIE \\
\hline Model 2 & {$[0: 0.3] \delta e=0.05$} & {$\left[0^{\circ}: 30^{\circ}\right] \delta i=1^{\circ}$} & {$\left[30^{\circ}: 110^{\circ}\right]+\left[250^{\circ}: 330^{\circ}\right] \delta \sigma=2^{\circ}$} & $50 \mathrm{Myr}$ & OSS & HYB \\
\hline Model 3 & {$[0: 0.3] \delta e=0.01$} & {$\left[0^{\circ}: 30^{\circ}\right] \delta i=5^{\circ}$} & {$\left[0^{\circ}: 360^{\circ}\right] \delta \sigma=2^{\circ}$} & $10 \mathrm{Myr}$ & OSS & HYB \\
\hline Model 4 & {$[0: 0.2] \delta e=0.01$} & $0^{\circ}, 30^{\circ}$ & {$\left[30^{\circ}: 110^{\circ}\right]+\left[250^{\circ}: 330^{\circ}\right] \delta \sigma=2^{\circ}$} & $50 \mathrm{Myr}$ & SJA, SJS, OSS & HYB \\
\hline
\end{tabular}

investigation of asteroids in stable chaotic motion (ASCs) or Karlsson (1984). Thus it is necessary to use different integration techniques and models to find out whether some dynamical effects are real or an artificial bias due to the influence of chaos. For this study, we used three different numerical integration methods:

- Lie integration method (LIE): this method is based on Lie Series and is very accurate. It has an adaptive stepsize and can handle close encounters and high values of eccentricity. For details see Lichtenegger (1984), Hanslmeier \& Dvorak (1984), or Asghari et al. (2004).

- Hybrid symplectic/Bulirsh-Stoer integrator (HYB): this is the hybrid routine from the Mercury 6 package by Chambers (1999). It is very fast but only moderately accurate and can also handle close encounters.

- Bulirsh-Stoer method (B-S): this is the general BulirshStoer routine from the Mercury 6 package by Chambers (1999). It is very accurate but slow. The results from this method were used as a "reference" to compare the validity of the other methods.

To analyze the data, the following two parameters were used:

- the escape time $\left(t_{\text {esc }}\right)$ - the time interval after which an asteroid is ejected from the system (i.e. when the eccentricity reaches values near unity) or leaves the Trojan cloud.

- the libration width $\Delta \sigma$ - the difference between $\sigma_{\max }$ and $\sigma_{\text {min }}$ with $\sigma=\lambda-\lambda_{\mathrm{JUP}}$, where $\lambda=\omega+\Omega+M$. Here $\sigma$ equals $60^{\circ}$ at point $L_{4}$ and $300^{\circ}$ at $L_{5}$.

The dynamical models used for this work are listed in Table 1. Only the elements $e, i$, and $\sigma$ (and thus the mean anomaly $M$ ) were changed, while the remaining initial orbital elements were set equal to that of Jupiter. We did not vary $a$ for two reasons: first, this has already been done by others (see Sect. 4), and second, it can be seen that the variation in $e$ and $i$ is much larger than the variation in $a$ by looking on the distribution of Trojan orbital elements. We neglected the inner planets in the integrations (but their mass was added to that of the Sun) and used only the outer ones (with their actual orbital elements obtained from the JPL Horizons database). We are aware that these initial conditions are not totally natural. Nevertheless, we decided to use these initial conditions because they were also used in the parameter studies by other authors (e.g. Dvorak \& Schwarz 2005; Pál \& Süli 2004) so that our findings can be compared to these works and can be used as useful extensions of their results. Also (see Sect. 5.6), the results are sound and in good agreement with existing data. Future work (see Sect. 6) will also include calculations with "natural" initial conditions (e.g. by using the proper elements).

According to the models, different dynamical configurations were used:

- OSS: the outer solar system (including Sun, Jupiter, Saturn, Uranus, and Neptune);
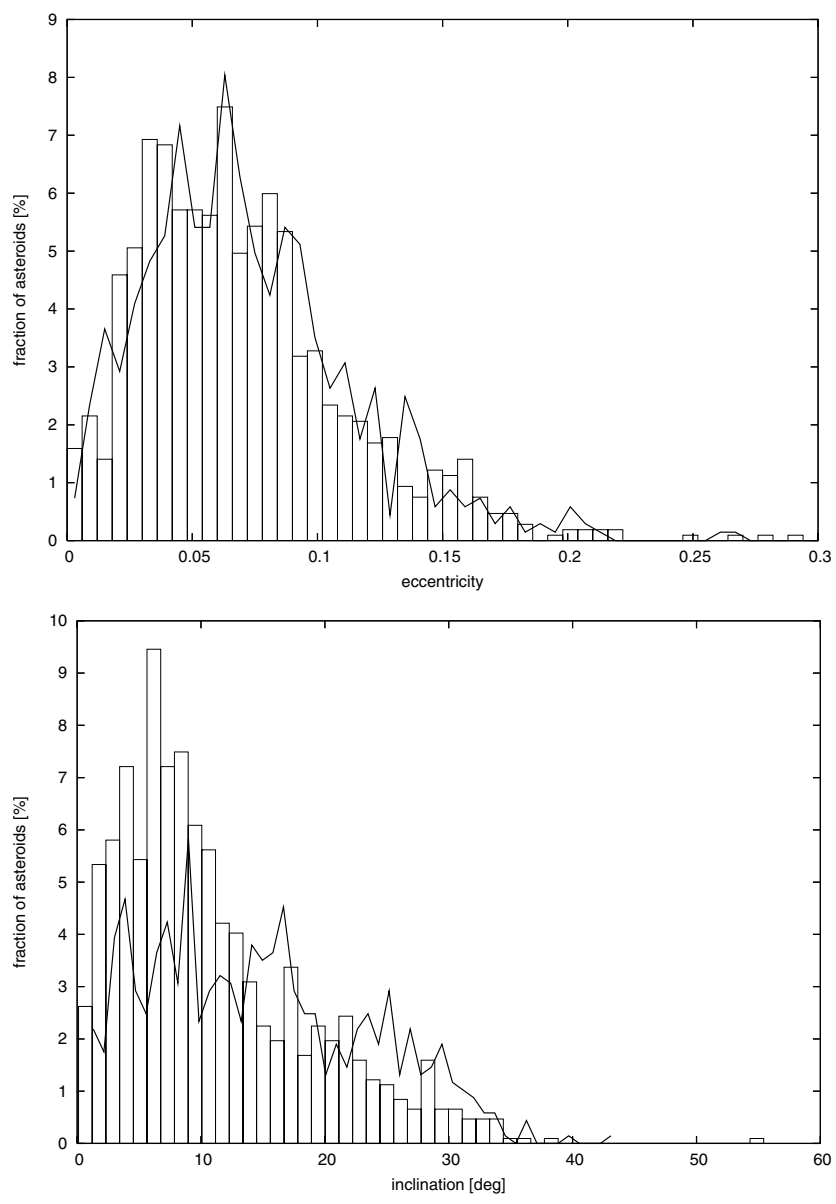

Fig. 1. Distribution of the real $L_{4}$ (bars) and $L_{5}$ (lines) Trojans (for 01.01.2005 00 UT) with respect to their eccentricities (top) and inclinations (bottom).

- SJS: including Sun, Jupiter, and Saturn;

- SJA: including only Sun and Jupiter (in fact, this model is equivalent to the elliptic, restricted three-body problem).

Two of the models were integrated for 50 Myrs - a time scale that is long enough to investigate the consequences of most of the dynamical effects (see e.g. Dvorak \& Schwarz 2005 or Tsiganis et al. 2000). The remaining two models were integrated for 10 Myrs to see how the size and shape of the stability regions already change on relatively short timescales.

\section{Stable librations around $L_{4}$ and $L_{5}$}

At first we want to investigate the motion in the direct vicinity of the Lagrangian points. Thus, in model 1 (see Table 1), we varied only the initial eccentricity (between 0 and 0.2 ) and the initial inclination (between $0^{\circ}$ and $30^{\circ}$ ). Figure 1 shows that this choice of initial conditions covers the variation in the elements of the real Trojans. The initial semimajor axis was set equal to that of Jupiter and the initial mean anomaly equal to the values 


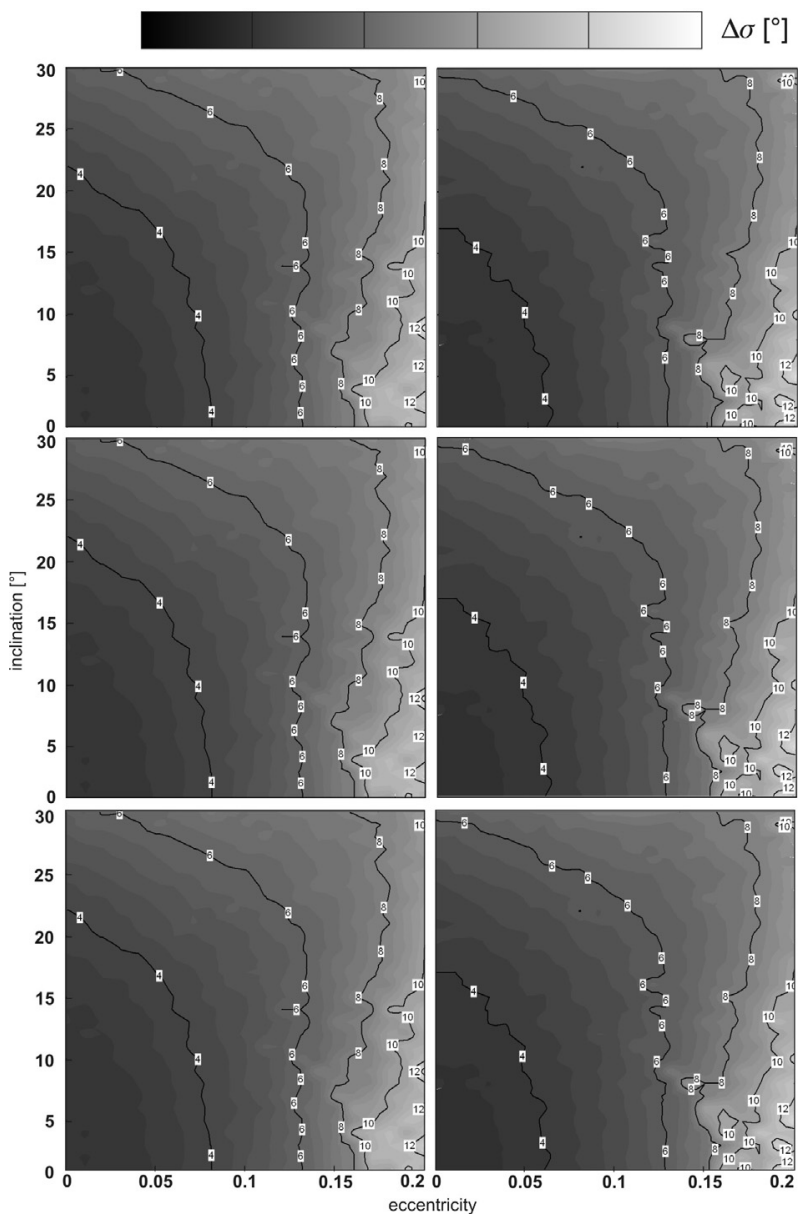

Fig. 2. Values of $\Delta \sigma$ in the $(e-i)$ plane for $L_{4}(l e f t)$ and $L_{5}$ (right) calculated with a Bulirsh-Stoer integrator (top), a hybrid integrator (middle), and using the Lie-Integration method (bottom).

of $L_{4}$ and $L_{5}$ such that the asteroids are always placed initially in the vicinity of the Lagrangian points. The integration time was $10^{7}$ years and all planets of the outer solar system (OSS) were used as perturbers. To test the validity of the three integration methods, all of them were used for this model.

Figure 2 shows the results. Here we plotted the value of $\Delta \sigma$ for the different initial values of $e$ and $i$. The first thing to notice is that there is no influence of the integration method - all three of them delivered the same output ${ }^{2}$. It can also be seen that $L_{4}$ and $L_{5}$ behave similarly, although there is a small region of larger libration $\left(\Delta \sigma \approx 12^{\circ}\right)$ near $i=5^{\circ}$ and $e=0.15$ that seems to be larger for $L_{5}$ than for $L_{4}$, which indicates that the dynamical properties of $L_{5}$ induce larger librations if the eccentricity is increased. It can also be seen that the region enclosed by the innermost contour-line - indicating libration-widths smaller than $4^{\circ}$ - is smaller for $L_{5}$ than for $L_{4}$ (see details in Sect. 5).

Figures 3 and 4 show the interaction between initial eccentricity, initial inclination, and libration around the Lagrangian points in more detail: in Fig. 3 it is shown that in general an increase of eccentricity also causes higher values of $\Delta \sigma$, independent of $i$. An interesting feature can be seen near values of the initial eccentricity of $e_{\text {crit }} \approx 0.15$ : here the lines for the different initial values of $i$ cross. That means that for initial

${ }^{2}$ In the following we will only present the pictures obtained with the hybrid integration method (HYB). Additional calculations have shown that all three methods give the same output; thus for all future integrations we have neglected the LIE and B-S methods.
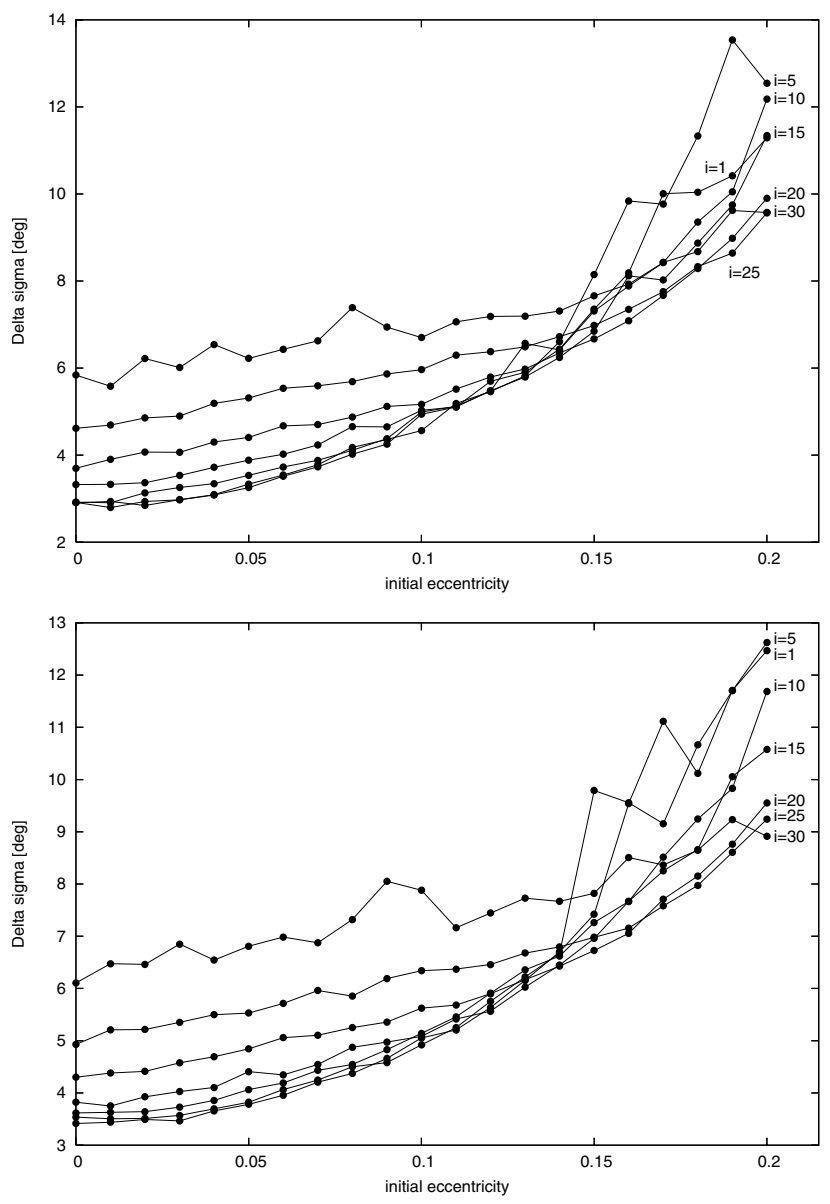

Fig. 3. Dependence of $\Delta \sigma$ on the eccentricity for $L_{4}$ (top) and $L_{5}$ (bottom) for Model 1 (HYB).

eccentricities lower than $e_{\text {crit }}$, low inclinations cause smaller librations around $L_{4}$ or $L_{5}$ and high inclinations cause larger librations, whereas for initial eccentricities that are higher than $e_{\text {crit }}$ lower initial inclinations cause larger librations (and vice versa). Thus, if a Trojan asteroid has an eccentricity higher than $e_{\text {crit }} \approx$ 0.15 , its inclination also has to be high to be protected from close encounters and thus ejection of the system. Figure 4 shows the same behavior: the width of libration increases with increasing values of the initial inclination when the initial eccentricity is lower than $e_{\text {crit }}$ and decreasing otherwise.

\section{Size of the stability region}

The size and structure of the stability region has been investigated by many authors in the past. Rabe in 1967 was one of the first to give an analytical boundary of stability based on the proper eccentricity and the libration amplitude $(\Delta \sigma)$. He showed that all known Trojans (in 1966) lie inside this region of stability $^{3}$. In 1997, Levison et al. performed extensive numerical integrations and showed that the region of effective stability on a timescale of $4.5 \mathrm{Gyr}$ - is smaller than that derived by Rabe. Robutel et al. (2005) use frequency analysis to investigate the stability of Trojans around $L_{4}$ in the $(a-e)$ plane (for the SJS model) and identify the main resonances that "form the dynamical skeleton and dictate the long-term dynamics of the Trojan

${ }^{3}$ For an initial value of $\Delta \sigma=0^{\circ}$, the stability region encloses all values of proper eccentricity that are lower than $\approx 0.21$; this value decreases to $\approx 0.1$ for an initial $\Delta \sigma$ of $43^{\circ}$. 

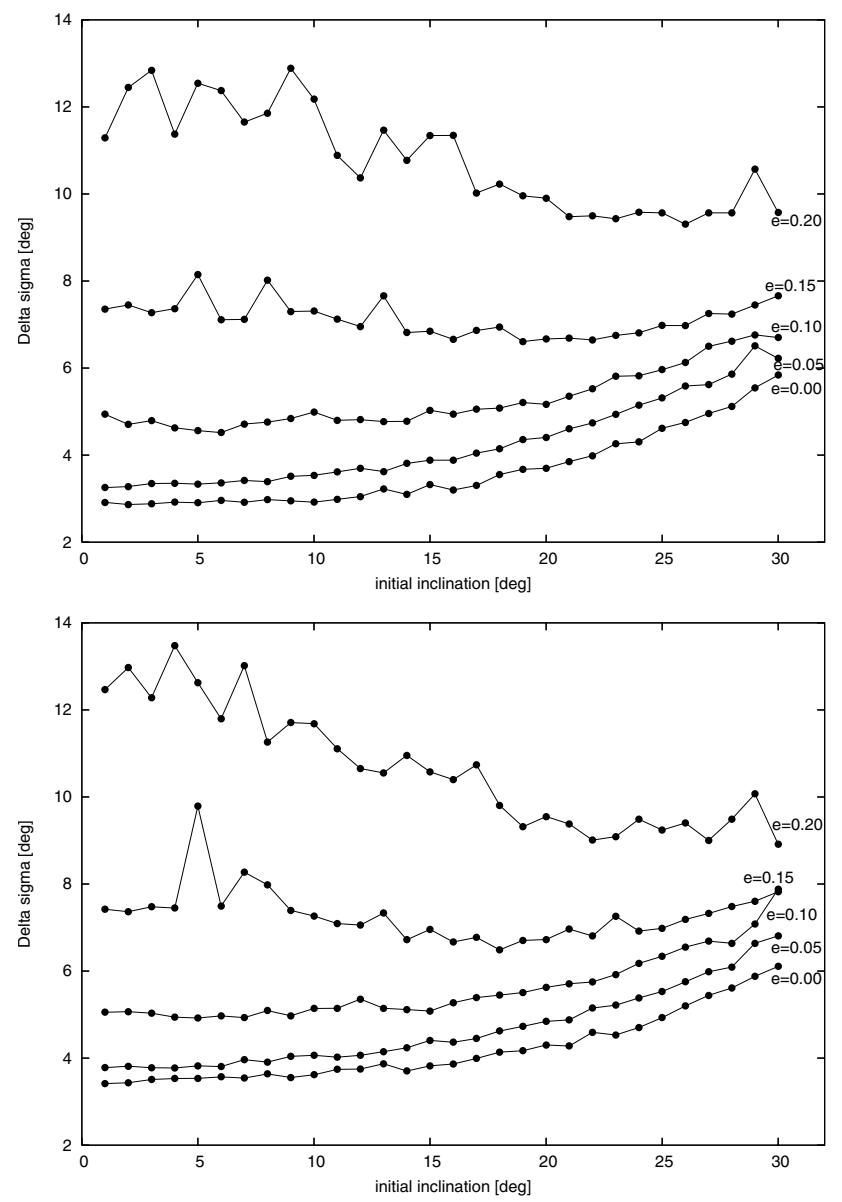

Fig. 4. Dependence of $\Delta \sigma$ on the inclination for $L_{4}$ (top) and $L_{5}$ (bottom) and different initial values of the eccentricity for Model 1 (HYB).

asteroids". Tsiganis et al. (2005) also researched the effective stability regions of the Trojan asteroids and found a power-law statistical correlation between the Lyapunov time and the escape time of Trojans on chaotic orbits. They also show that the regions of effective stability contain bodies that become chaotic. Dvorak \& Schwarz (2005) investigated the size of the stability regions around the Lagrangian points in the $(a-\sigma)$ plane and mapped the stability region by using the Lyapunov characteristic exponents (LCEs). Since two of the aforementioned works (Dvorak \& Schwarz 2005; Robutel et al. 2005) already deal with the dependence of the size of the stability region on the variation in the semimajor axis, we restricted this work to an investigation of the dependence on eccentricity and inclination.

\subsection{The influence of eccentricity}

Figure 5 shows the results from the integration of model 2. In this model also $\sigma$ was varied and thus the test particles are now spread out along the orbit of Jupiter in the neighborhood of the Lagrangian points unlike in model 1 . Additionally, the integration time was increased to $5 \times 10^{7}$ years. The contour plots give the value of the escape time in its dependence on the initial inclination and $\sigma$ values. The first thing to notice is the asymmetry in the $\sigma$ values: the stable zone extends unevenly wide around the position of the Lagrangian points. For values lower than $60^{\circ}\left(L_{4}\right)$ (or higher than $300^{\circ}\left(L_{5}\right)$ ) the orbits are less stable than in the other direction (note that Jupiter has $\sigma=0^{\circ}$ ). For low eccentricities $\left(e_{\mathrm{INI}}=0.00\right.$, Fig. 5 top), there are almost no unstable orbits;
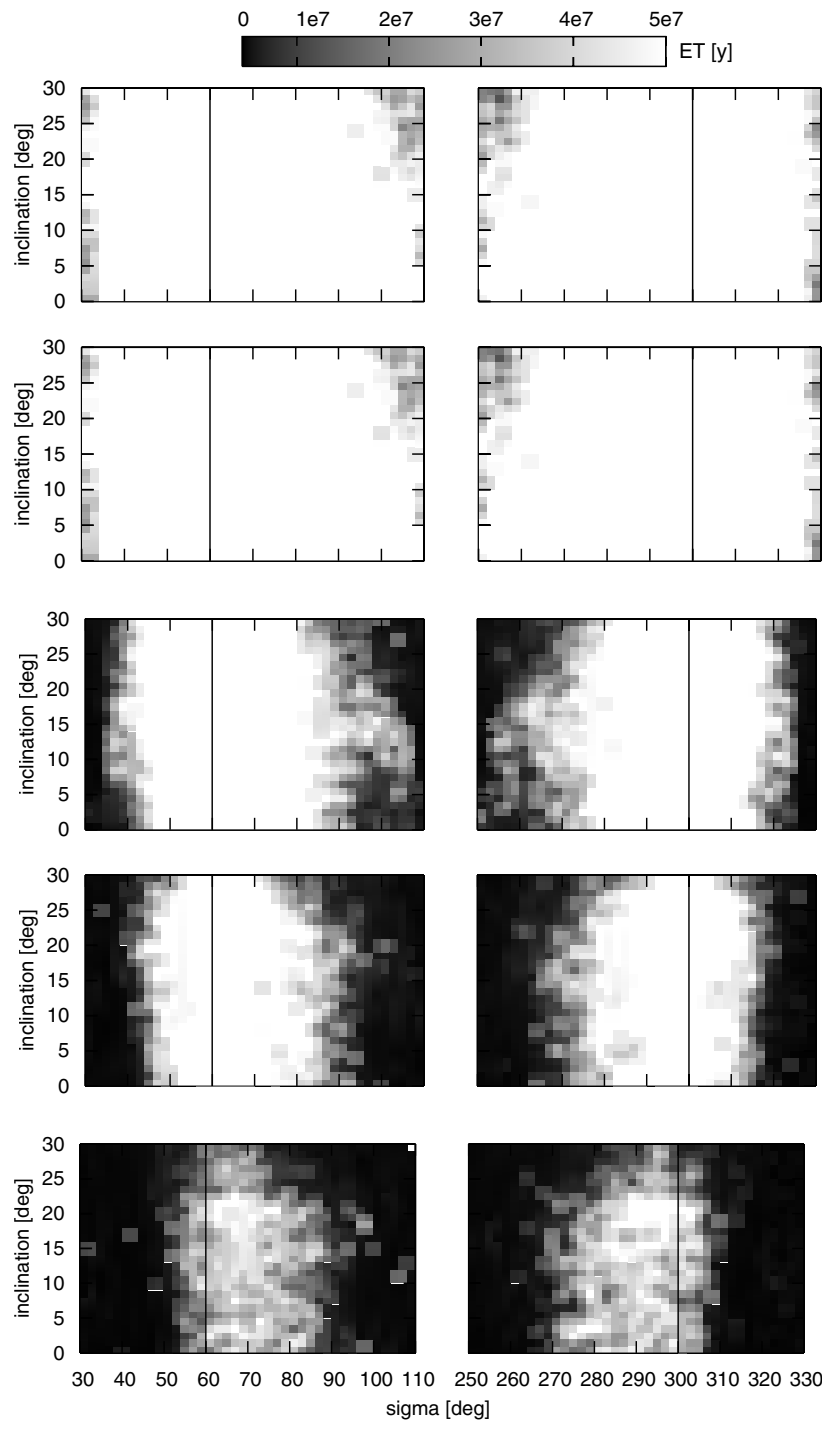

Fig. 5. Extension of the stability regions in the $(\sigma-i)$ plane around $L_{4}$ (left) and $L_{5}(r i g h t)$ for an initial eccentricity of $e_{\mathrm{INI}}=0($ top $)$, $e_{\mathrm{INI}}=0.1$ (second row), $e_{\mathrm{INI}}=0.15$ (third row), $e_{\mathrm{INI}}=0.2$ (fourth row), and $e_{\mathrm{INI}}=0.3$ (bottom): dark colors show unstable motion; light colors denote stable motion. The vertical lines indicate the position of the Lagrangian points; the integration was performed in the OSS model.

also the plots for $e_{\mathrm{INI}}=0$ and $e_{\mathrm{INI}}=0.05$ are almost identical; we have omitted the figure for $e_{\mathrm{INI}}=0.05$. The size of the stable region around the Lagrangian points decreases for higher values of e (Fig. 5 second and third row, $e_{\mathrm{INI}}=0.10$ and $e_{\mathrm{INI}}=0.15$ ). Here the difference between the two "directions" can be seen very clearly: in the direction towards Jupiter, the border of the stable region shows almost no changes with the initial inclination, whereas towards the opposite direction to Jupiter the border of stability decreases when the initial inclination increases (Fig. 5, second row) or becomes very diffuse (Fig. 5, third row). In Fig. 5 (third row) a "bulge"-like structure is visible for inclinations around $8^{\circ}-15^{\circ}$ and $\sigma>90^{\circ}$ (or smaller than $270^{\circ}$ ) where the stability region has its largest extension, corresponding to the distribution of actual inclinations in Fig. 1, where the maximum also lies in this region. Figure 5 (fourth row) $\left(e_{\mathrm{INI}}=0.20\right)^{4}$ shows the further decrease in the size of the stable region, and it

\footnotetext{
${ }^{4}$ We have omitted the figure for $e_{\mathrm{INI}}=0.25$ because it shows no new information.
} 

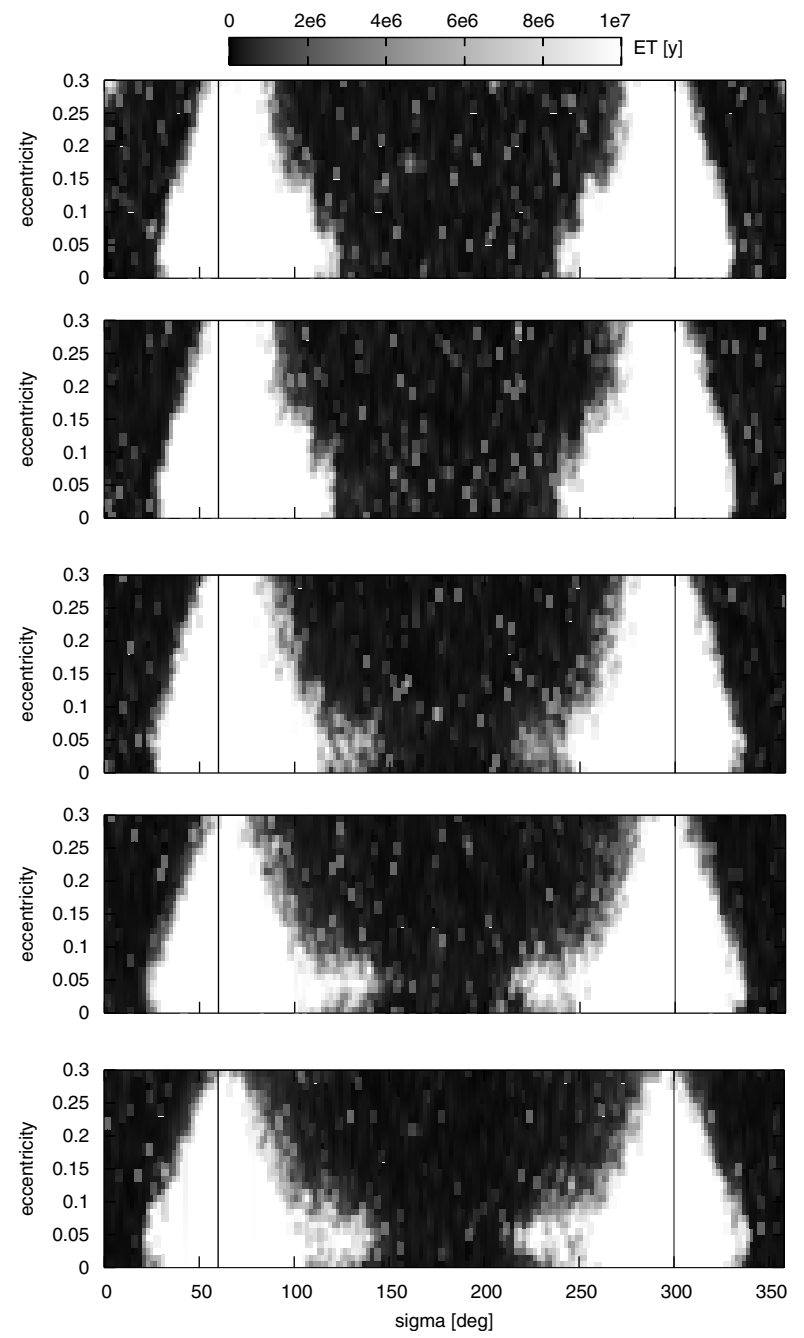

Fig. 6. Stability region in the $(\sigma-e)$ plane for an initial inclination of $i_{\mathrm{INI}}=0^{\circ}($ top $), i_{\mathrm{INI}}=10^{\circ}$ (second row $), i_{\mathrm{INI}}=20^{\circ}$ (third row $), i_{\mathrm{INI}}=25^{\circ}$ (fourth row), and $i_{\mathrm{INI}}=30^{\circ}$ (bottom): dark colors show unstable motion; light colors denote stable motion. The vertical lines indicate the position of the Lagrangian points at $60^{\circ}\left(L_{4}\right)$ and $300^{\circ}\left(L_{5}\right)$; the integration was performed in the OSS model.

can again be observed that the largest extension of the stability region lies at medium values of inclination For an initial eccentricity of $e_{\mathrm{INI}}=0.30$ (Fig. 5 , bottom), almost no stable orbits are left ${ }^{5}$. In the direction towards the position of Jupiter, the region of stability has more or less disappeared; the only place left for stable motion is towards the region opposite to Jupiter and for higher values of inclination.

\subsection{The influence of inclination}

Figure 6 shows the results from the integration of model 3 . The contour-plots show the value of the escape time depending on the initial values of the eccentricity and $\sigma$ which are different from model 2, where only the extended neighborhood of the Lagrangian points was investigated. Here the whole range $\sigma=\left[0^{\circ}, 360^{\circ}\right]$ was integrated. It can be seen in Fig. 6 that the shape of the stable region is now different from the stable region of model 2: now the borders on both sides (towards the

5 Note that for high eccentricities the position of Lagrangian points differs from $\sigma=60^{\circ}$ and $\sigma=300^{\circ}$. Strictly speaking, these values are only valid for the restricted (circular) three-body problem.
Table 2. Percentage of stable orbits for model 3.

\begin{tabular}{lccc}
\hline \hline$i_{\mathrm{INI}}[]$ & total & $e \leq 0.15$ & $e>0.15$ \\
\hline 0 & 33.42 & 43.66 & 22.52 \\
5 & 33.85 & 44.60 & 20.98 \\
10 & 34.55 & 45.13 & 23.30 \\
15 & 34.66 & 43.75 & 23.40 \\
20 & 34.55 & 44.38 & 22.57 \\
25 & 32.62 & 45.15 & 18.09 \\
30 & 30.90 & 44.93 & 14.93 \\
\hline
\end{tabular}

direction of Jupiter and towards the opposite direction) are inclined. But the dependence of the limiting value of $\sigma$ on the initial eccentricity is less steep for the direction towards Jupiter. Another remarkable feature can be seen by comparing the region above values of $e_{\mathrm{INI}} \approx 0.15$ with the one below: the borders of the upper region show very little dependence on the initial eccentricity and are almost constant, whereas the borders of the lower region vary in the way described above.

This upper region remains almost constant in shape and size until $i_{\mathrm{INI}} \geq 25^{\circ}$ where it starts shrinking (see Table 2 for details). In this model the "critical" eccentricity $e_{\text {crit }} \approx 0.15$ plays a different role than in model 1 (see Sect. 3). There, all test particles were placed in the vicinity of the Lagrangian points (no variation of $\sigma$ ) and a high inclination caused smaller perturbations than a low inclination when $e>e_{\text {crit }}$ (and vice versa). In model 3 (where we varied $\sigma$ from $0^{\circ}$ to $360^{\circ}$ ), a high initial inclination is now no longer a protection against perturbations, and it causes more unstable orbits than a low initial inclination. Nevertheless the stability region above $e \approx e_{\text {crit }}$ shows the abovementioned different behavior under the influence of inclination.

The behavior of the lower region is more complex. No dramatic change is visible until $i_{\mathrm{INI}}=20^{\circ}$. At this value the stable region starts to "intrude" the region opposite to Jupiter near the line $e_{\mathrm{INI}} \approx 0.05$. This process continues as $i_{\mathrm{INI}}$ becomes larger, although the total percentages of stable orbits in the region $e_{\mathrm{INI}}<0.15$ remains more or less constant. Figure 1 shows that the maximum of the eccentricity distribution of real Trojans also lies around $e \approx 0.05$.

\subsection{The influence of the planets}

To see how the outer planets influence the size of the stability region, all three different sets of perturbing bodies were used for integration (OSS, SJS, and SJA) in model 4. For all three cases, the stability region in the $(\sigma-e)$ plane was integrated for two different values of initial inclination $\left(i=0^{\circ}\right.$ and $\left.i=30^{\circ}\right)$. Figure 7 shows the contour plots for $L_{4}{ }^{6}$. It is clear that, in the absence of the perturbation of all other outer planets, the SJA model will show a large amount of stable orbits; the two regions of instability are placed at higher eccentricities and far away from the position of the Lagrangian point. Figure 7 (bottom left) again shows the asymmetry concerning the different directions (towards Jupiter or towards the opposite direction) caused by the configuration of the system. Whereas Trojans placed in the region near Jupiter $\left(\sigma_{\text {INI }} \approx 30^{\circ}\right)$ are stable only for low initial eccentricities $\left(e_{\mathrm{INI}}<0.07\right)$, the Trojans placed in the opposite region $\left(\sigma_{\mathrm{INI}} \approx 110^{\circ}\right)$ are stable up to initial eccentricities of $e_{\mathrm{INI}} \approx 0.15$.

In Fig. 7 (bottom right) the initial inclination was increased to $30^{\circ}$ and now there is not much difference between the two

\footnotetext{
6 The behavior of $L_{5}$ was not different from $L_{4}-$ thus we omit the corresponding figure.
} 


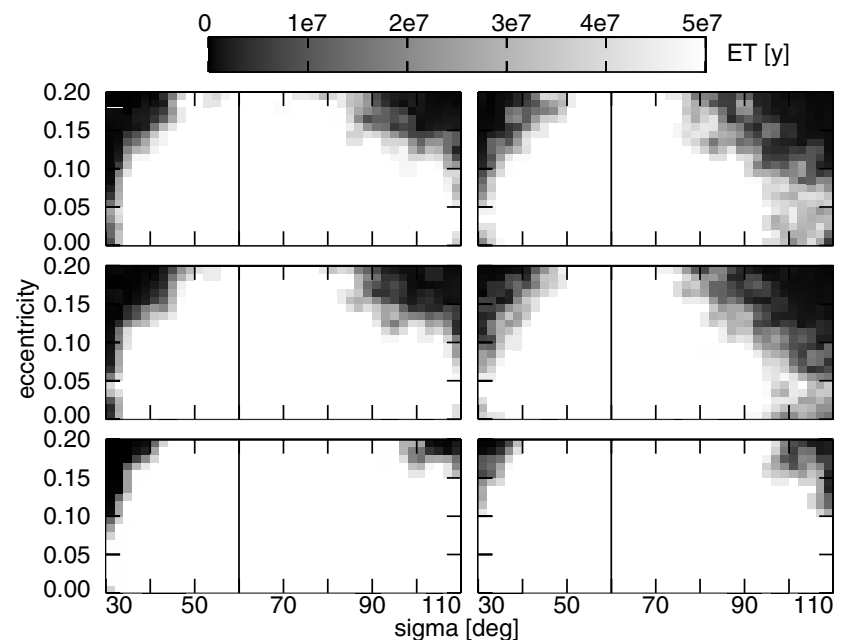

Fig. 7. Stability region in the $(\sigma-e)$ plane around $L_{4}$ for the OSS model (top), the SJS model (middle), and the SJA model (bottom). The left column shows the stability region for an initial inclination of $i_{\mathrm{INI}}=0^{\circ}$; the right column for $i_{\mathrm{INI}}=30^{\circ}$. The vertical lines indicate the position of the Lagrangian point.

regions. On both "sides" stable orbits have to have an initial eccentricity lower than $e_{\mathrm{INI}}=0.10$ although the instability region for high eccentricities is smaller in the region near Jupiter. Figure 7 (top and middle) shows no serious difference, so Uranus and Neptune not seem to have any strong influence on the dynamics of Trojans (at least over the timescale of this integration!). Therefore it is also reasonable to conclude that any dynamical difference between the Lagrangian groups $L_{4}$ and $L_{5}$ is due to the perturbations of Saturn. This topic will be investigated in the next section.

\section{The difference between the two Trojan populations}

One important fact that has not yet been explained completely is the difference between the populations of $L_{4}$ and $L_{5}$ Trojans (see Fig. 8 that shows how the number of known Trojans increases during the past years). There are several possible explanations for this phenomenon:

1. Observational reasons: the asteroids in $L_{5}$ could be smaller, darker, or have higher inclinations than in $L_{4}$ and thus are more difficult to observe.

2. Different formation processes: if the formation processes in the vicinity of $L_{4}$ had better conditions, there would be more asteroids than in $L_{5}$. It could also be possible that asteroids are more likely captured near $L_{4}$ than $L_{5}$.

3. Catastrophic event: a catastrophic event during the formation of the Solar System could be responsible for the smaller number of $L_{5}$ asteroids.

4. Chaotic capture: recent works have shown that the Trojan asteroids may have been captured by Jupiter and that this process shows chaotic features that could be responsible for the asymmetry.

5. Dynamical reasons: due to the gravitational influence of the other large planets, the region around $L_{4}$ could be more stable than the one around $L_{5}$. The difference could also be due to non-gravitational forces that act differently on the two Lagrangian points.

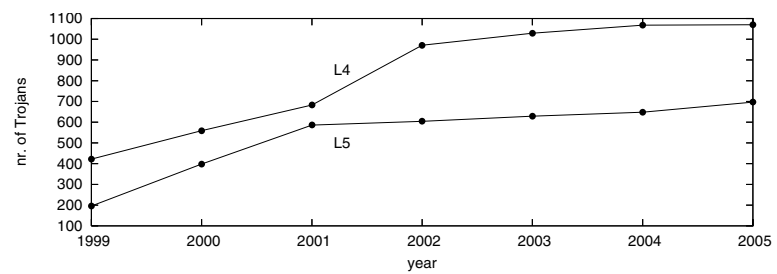

Fig. 8. Number of Trojan asteroids discovered near $L_{4}$ and $L_{5}$ in the years 1999-2005. During the past years, the fraction of the two groups was always near $\frac{5}{3}$.

Points 1), 3), and 4) are not the subject of this work; also, to investigate point 2) one needs different dynamical models than the ones used here. Thus this work will only deal with point 5). Nevertheless, since we were not able to find a review article on this topic, we want to try to summarize the existing results before presenting the results of this work. Because of the many effects that can be responsible for the difference in the Trojan population, one has to be very careful when interpreting the results. Thus, to ensure that our interpretation of the existing works is not biased in any sense, instead of summarizing the results in our own words, we want to cite the major points of the existing works as direct quotations.

\subsection{Observational reasons}

Shoemaker et al. (1989) present an observational reason for the difference. They wrote: "It appears to be an accident of history that the L4 region has been more thoroughly explored". Because in that time the $L_{5}$ region was located on the sky near the Milky Way, observational conditions were not equal to that of $L_{4}$; thus, this was thought to be the reason for the asymmetry. This argument was also cited in later years in (2000) by Fleming \& Hamilton and by Kuchner et al. (2000) as a reason for the difference between the number of observed asteroids. But Fig. 8 shows that the difference between the two populations is still present although many new discoveries have been made in recent years. The number of observed Trojans around the $L_{4}$ equilibrium point is still significantly larger than around $L_{5}$, with the fraction between these two groups close to $\frac{5}{3}$. The reason could also be a higher inclination of the $L_{5}$ Trojans that make them more difficult to discover. In fact Fig. 1 shows that the percentage of known $L_{5}$ Trojans with high inclinations is higher than the one for $L_{4}$. Additionally, there could be a difference in the size distribution of the two groups that causes a different probability of observation. But the size distribution of Trojans is still not fully understood (see e.g. Marzari et al. 1996, 1997 or Tsiganis et al. 2005). Also, the present size distribution is closely connected to the formation process of Trojans.

\subsection{Different formation processes}

Peale (1993) searched for the reason for the difference by investigating the formation of Trojans. He simulated the evolution of Trojan precursors in the primordial solar nebula and found an asymmetric distribution of Trojan asteroids due to the eccentricity of Jupiter. But his results showed that the stability of bodies around $L_{5}$ is increased, while the stability of bodies around $L_{4}$ is decreased, which is opposite to the situation we observe today. He argues that the collisional evolution of the Trojans from the time of the formation until today "may have skewed the mass distribution in such a way as to mask what was there initially". 
This result was confirmed by Marzari \& Scholl (1998), who write that "According to this asymmetry, we expect that more small planetesimals are captured in L5 than in L4 in the earlier stages of Trojan evolution". Thus, the subsequent collisional evolution would show more impacts in $L_{5}$, and this higher impact rate could be the reason for the asymmetry. There seem to be indications that the subsequent collisional evolution of Trojan asteroids indeed favors the $L_{4}$ group. In 2001, Beaugé \& Roig used semi-analytical models and identified several asteroidal families and clusters around $L_{4}$ and $L_{5}$. They write that "The clusters detected in $L_{5}$ are much less significant than those in $L_{4}$, even allowing for the difference in the base population".

\subsection{Catastrophic event}

Up to now we know of no work that has investigated the special case of a catastrophic event that may have depleted the $L_{5}$ population sometime during the evolution of the Solar System. Nevertheless, this could be a possible reason. It is known that collisions between protoplanets have happened during the formation of the Solar System and also that several larger protoplanets (comparable to the Earth) were ejected from the outer Solar System (see e.g. Goldreich et al. 2004). Such an escaping protoplanet could have come close to the $L_{5}$ group and subsequently induced an ejection of small bodies.

\subsection{Chaotic capture}

A recent work by Morbidelli et al. (2005) investigates the possible capture of Trojan asteroids by Jupiter. It is suggested that the asteroids formed in more distant regions and then, during the time when the giant planets migrated inwards, were captured by Jupiter in the Trojan-type motion. This capture was only possible during a short time span, just after Jupiter and Saturn crossed their mutual 1:2 resonance. During this time, the dynamics of the Trojan region were completly chaotic. Their simulations reproduce the orbital distribution of the Trojans, and the chaotic process of capture may also account for the difference between the $L_{4}$ and $L_{5}$ populations.

\subsection{Dynamical reasons}

One of the first who tried to find a dynamical solution was Barber (1986). He investigated the influence of Saturn and found "little difference" between the two groups. Only in one special case for inclinations near $5^{\circ}$, an eccentricity near $e=0.05$, and libration amplitudes between $20^{\circ}$ and $40^{\circ}$ - did he find that $L_{5}$ Trojans are less stable than $L_{4}$ Trojans when one includes Saturn in the model. However, the integration time of his model was only one million years. Additionally only a relatively large time-step could be used and thus " $[. .$.$] the truncation error became impor-$ tant". He concludes " [...] any conclusion that Saturn is responsible for the $L_{4} / L_{5}$ differentiation must be tentative indeed". If we check model 2 of our work (see Fig. 5) for $e_{\text {INI }}=0.05$, we find that at $i_{\mathrm{INI}}=5^{\circ}$ all orbits are stable for $L_{4}$ (41 out of 41) and 38 out of 41 are stable for $L_{5}$. Thus in this special case, $L_{5}$ is more unstable than $L_{4}$, but for any decisive conclusion a larger sample has to be checked.

Recently, several new dynamical studies were performed to identify a possible difference due to the perturbation of Saturn. Pál \& Süli (2004) investigated the stability regions around the Lagrangian points in the $(a-e)$ plane and for the non-singular elements $(h-k)$. As an indicator of stability they did not
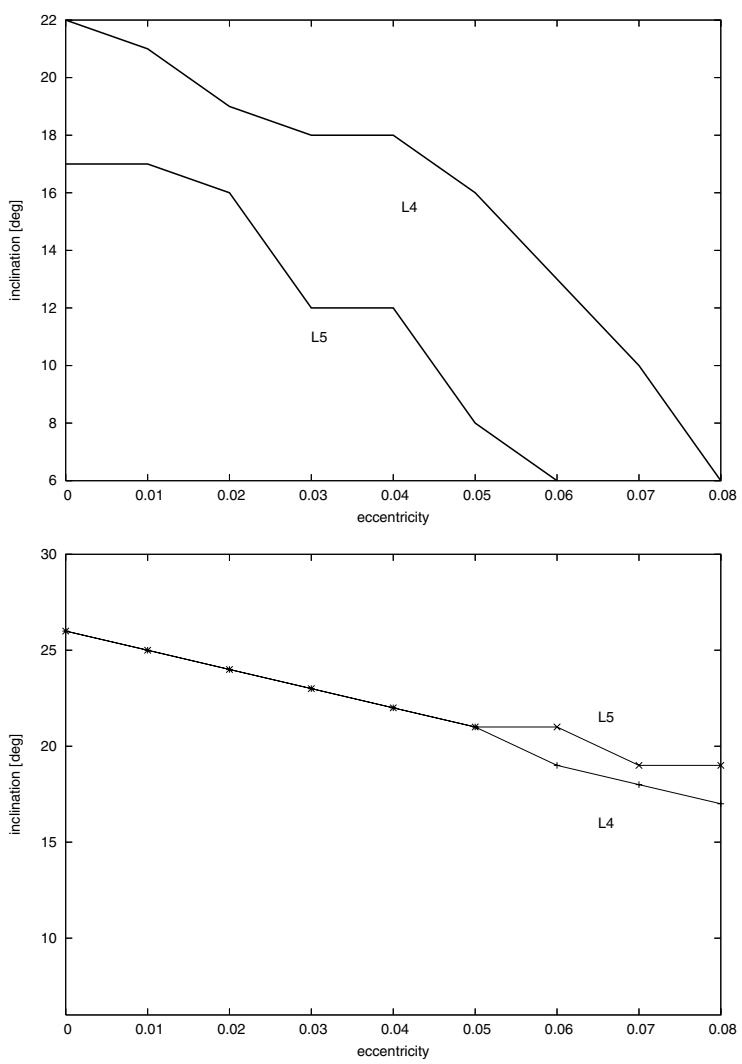

Fig. 9. Top: borders of the region where the libration width $\Delta \sigma$ of the Trojans is smaller than $4^{\circ}$ (Model $1-\mathrm{HYB}$ ). The region for $L_{5}$ is clearly smaller. Bottom: same in the SJA model. No significant difference can be seen.

directly use the orbital elements but rather several chaos indicators, namely the Fourier spectra numbers (FSN) and Lyapunov characteristic exponent (LCE). Their plots "[...] show clearly that the size of the stable region around the two Lagrangian points is smaller around the trailing point". But there were differences between their calculated mean values of the longitude of the perihelion and the observed one, which indicates some problems with the fitting procedure and the determination of the stable regions. Schwarz et al. (2004) and Dvorak \& Schwarz (2005) also present some numerical indications (based on the evaluation of Lyapunov exponents) that the difference between the numbers of $L_{4}$ and $L_{5}$ Trojans is indeed due to a dynamical effect and not an observational bias. But they also conclude that the results - found with the aid of the LCE - could be biased by the choice of initial conditions.

\subsection{Results}

In this subsection we now want to summarize the results of our calculations concerning the difference between the two Trojan groups. We want to emphasize again that we rely only on the evolution of the actual orbital elements, not on any chaos indicator. Chaos indicators are a very important and valuable tool; but since it is still not clear if the reason for the difference between $L_{4}$ and $L_{5}$ is dynamical, we are reluctant to use it here because the application of e.g. Lyapunov characteristic numbers could cause a bias in the results (see Pál \& Süli 2004).

Figure 9 (left) shows some results of the model 1 (HYB, OSS) integration. We show the borders of the region where the libration width $\Delta \sigma$ is smaller than $4^{\circ}$. This value was chosen 
Table 3. Percentage of stable orbits for model 2.

\begin{tabular}{lcc}
\hline \hline$e_{\mathrm{INI}}$ & $L_{4}$ & $L_{5}$ \\
\hline 0.00 & 94.02 & 93.39 \\
0.05 & 94.10 & 92.29 \\
0.10 & 80.96 & 79.70 \\
0.15 & 57.83 & 57.36 \\
0.20 & 43.27 & 41.70 \\
0.25 & 29.19 & 30.21 \\
0.30 & 11.96 & 14.56 \\
\hline
\end{tabular}

because we want to investigate the stability in the direct vicinity of Lagrangian points. A small libration width ensures that the asteroid always stays near the Lagrangian point. The value of $4^{\circ}$ is somewhat arbitrary, but turned out to be good choice ${ }^{7}$. It is clearly visible that the abovementioned region is smaller around the Lagrangian point $L_{5}$ than around $L_{4}$. Before we draw some conclusions from this fact, we want to summarize again some properties of the integration that was performed in this case (model 1):

1. The test asteroids of this model were placed in the vicinity of $L_{4}\left(L_{5}\right)$; i.e. the initial value of the mean anomaly $M$ was always $M_{\mathrm{JUP}}+60^{\circ}\left(M_{\mathrm{JUP}}-60^{\circ}\right)$. Only the initial eccentricity and initial inclination were varied according to the distribution of the real asteroids.

2. Compared to the other models, the integration time was relatively short (10 Myrs).

Thus, because of 1), the results of this model can be used to describe the direct vicinity of the Lagrangian points, in contrast to the other models, where large areas surrounding Jupiters orbit were investigated. The libration width describes the amplitude an asteroid has when it is moving around the Lagrangian points. In this case, it indicates the maximum separation a body had from the Lagrangian point during the integration. Figure 9 (top) now shows that there are almost twice as many asteroids with very small amplitudes $\left(<4^{\circ}\right)$ around $L_{4}$ than $L_{5}$ - already on the relatively short timescale of 10 Myrs! Since the two Lagrangian points are dynamically equivalent in the restricted three-body problem and since the results described in the former section showed that the influence of Uranus and Neptune is negligible, this is a strong indication that any dynamical difference between the two Lagrangian points is due to the influence of Saturn. To check this assumption, we repeated the integration for the SJA model, now only including the Sun and Jupiter. Figure 9 (bottom) shows that now the two regions are almost identical. Thus we can confirm that any dynamical difference must indeed be due to the influence of Saturn!

The situation becomes more complicated when we take longer time scales and different initial conditions into account. Tables 3 and 4 show the numbers of stable asteroids in models 2 and 3 . We see that for model 2 the percentage of stable bodies around $L_{4}$ and $L_{5}$ is comparable, although the numbers are higher for $L_{4}$ (except for very high initial eccentricities) by $\approx 1 \%$. This difference is too small to make any conclusions on a probable dynamical difference between the two Lagrangian points. We find a similar behavior for model $3^{8}$ : the difference between the two values is even less.

We should also note that the fact that there seem to be slightly more stable asteroids around $L_{5}$ than $L_{4}$ for higher initial

\footnotetext{
${ }^{7}$ Note that we have obtained similar results for $3^{\circ}$ or $5^{\circ}$.

8 The percentage gives the number of stable orbits in between $\sigma=$ $\left[0^{\circ}, 120^{\circ}\right]$ for $L_{4}$ and $\sigma=\left[240^{\circ}, 360^{\circ}\right]$ for $L_{5}$.
}

Table 4. Percentage of stable orbits for model 3.

\begin{tabular}{lcc}
\hline \hline$i_{\mathrm{INI}}[\mathrm{o}]$ & $L_{4}$ & $L_{5}$ \\
\hline 0 & 49.89 & 49.30 \\
5 & 50.00 & 50.48 \\
10 & 51.67 & 51.02 \\
15 & 51.56 & 51.99 \\
20 & 51.08 & 50.81 \\
25 & 46.40 & 47.04 \\
30 & 44.25 & 44.19 \\
\hline
\end{tabular}

inclinations could support the thesis that the difference is due to observational reasons. If there were more $L 5$ Trojans with highly inclined orbits, they would be more difficult to observe than in $L_{4}$.

In the case of model 2, the integration time (50 Myrs) was five times longer than for model 3 (10 Myrs). But in both cases, we see no real difference between the stability of the two Lagrangian points. Thus, the fact that there is a difference for model 1 and none for models 2 and 3 is due not to the integration time but to the choice of initial conditions (although we suspect that a difference would appear if one would increase the integration time considerably).

\section{Conclusions}

The main goal of this work was to investigate the shape and size of the stability regions around the Lagrangian points $L_{4}$ and $L_{5}$ of the Sun-Jupiter system. This was done by investigating the results of several different numerical integrations. We investigated the libration around the Lagrangian points (model 1), show the dependence of the size and shape of the stability region on the initial eccentricity in the $(\sigma-i)$ plane (model 2$)$ and of the initial inclination in the $(\sigma-e)$ plane (model 3$)$, and investigated the influence of the perturbing planets Neptune, Uranus, Saturn, and Jupiter (model 4).

- We find that a "critical initial eccentricity" $e_{\text {crit }} \approx 0.15$ exists that determines the behavior of the stability region. In model 1 it gives the "border" where the influence of the initial inclinations changes from destabilizing to stabilizing behavior. In the $(\sigma-e)$ plane this initial eccentricity separates two regions: for $e_{\mathrm{INI}} \leq 0.15$ the percentage of stable orbits stays almost constant with increasing initial inclination, whereas for $e_{\mathrm{INI}}>0.15$ the percentage of stable orbits decreases with increasing initial inclination. In the $(\sigma-i)$ plane we observe a "bulge-like" structure for values around $e_{\mathrm{INI}} \geq 0.15$.

- The results from the models were also used to check if a dynamical reason exists for the difference between the number of asteroids observed in the $L_{4}$ and $L_{5}$ groups. We found a different dynamical behavior due to Saturn for model 1 but not for models 2, 3, or 4. This is explained as follows. The initial conditions for models 2 and 3 were chosen such that the stability region around the Lagrangian points and its borders could be mapped in full detail; therefore we placed test particles at many different values of the initial mean anomaly $M$. In model 1 all test particles were initially located close to the Lagrangian points $\left(M_{\mathrm{INI}}=M_{\mathrm{JUP}} \pm 60^{\circ}\right)$. The initial conditions for eccentricity and inclination were chosen in such a way as to resemble the distribution of real Trojans. Thus model 1 best describes the dynamical situation in the vicinity of the Lagrangian points where the real Trojan asteroids also move, whereas the initial conditions of 
models 2 and 3 also cover large areas where no real Trojans are observed. The difference in model 1 was found by direct analysis of the time evolution of orbital elements (not using any chaos indicators that could have biased the results); it is large and distinctive - thus we can conclude that this effect is indeed due to the influence of Saturn and not a numerical artefact.

The results of this work give rise to some interesting topics and questions that will be investigated in a future work:

- What is the underlying reason for the observed "bulge-like" structures in models 2 and 3? An analysis of the secular and mean-motion resonances will be needed to clarify these patterns.

- How does the motion of Trojans depend in detail on the value of $e_{\text {crit }} \approx 0.15$ ? New numerical integrations near this initial value of eccentricity are necessary.

- Is the reason for the difference in the numbers of observed Trojans near $L_{4}$ and $L_{5}$ indeed a dynamical one, due to the influence of Saturn? The results of this work support these theses but are not conclusive enough. New integrations for very long timescales and for carefully chosen sets of initial conditions will be performed.

Acknowledgements. Part of the numerical integrations were accomplished on the NIIDP (National Information Infrastructure Development Program) supercomputer in Hungary. We want to thank Dr. Rudolf Dvorak and Dr. Richard Schwarz for valuable comments and Dr. Áron Süli for assistance with the NIIDP supercomputer.

\section{References}

Asghari, N., Broeg, C., Carone, L., et al. 2004, A\&A, 426, 353 Barber, G. 1986, in Asteroids, comets, meteors II, ed. C. I. Lagerkvist, B. A. Lindblad, H. Lundstedt, \& H. Rickman, 161

Beaugé, C., \& Roig, F. 2001, Icarus, 153, 391

Bien, R. 1980, The Moon and the Planets, 22, 163

Bien, R., \& Schubart, J. 1984, CMDA, 34, 425

Brasser, R., Heggie, D. C., \& Mikkola, S. 2004, CMDA, 88, 123

Celletti, A., \& Giorgilli, A. 1991, CMDA, 50, 31

Chambers, J. 1999, MNRAS, 304, 793

Dvorak, R., \& Tsiganis, K. 2000, CMDA, 78, 125

Dvorak, R., \& Schwarz, R. 2005, CMDA, 92, 19

Efthymiopoulos, Ch. 2004, CMDA, 92, 29

Érdi, B. 1981, CMDA, 24, 377
Érdi, B. 1984, CMDA, 34, 425

Érdi, B. 1988, CMDA, 43, 303

Érdi, B. 1996, CMDA, 34, 425

Érdi, B. 1997, CMDA, 65, 149

Flemming, H., \& Hamilton, D. 2000, Icarus, 148, 479

Giorgilli, A., \& Skokos, C. 1997, A\&A, 317, 254

Goldreich, P., Lithwick, Y., \& Sari, R. 2004, ApJ, 614, 497

Hanslmeier, A., \& Dvorak, R. 1984, A\&A, 132, 203

Karlsson, O. 2004, A\&A, 413, 1153

Kuchner, M. J., Reach, W. T., \& Brown, M. E. 2000, Icarus, 145, 44

Levison, H., Shoemaker, E., \& Shoemaker, C. 1997, Nature, 385, 42

Lichtenegger, H. 1984, CMDA, 34, 357

Marzari, F., Scholl, H., \& Farinella, P. 1996, Icarus, 119, 192

Marzari, F., Farinella, P., Davis, D. R., Scholl, H., \& Campo Bagatin, A. 1997, Icarus, 125,39

Marzari, F., \& Scholl, H. 1998, Icarus, 131, 41

Marzari, F., \& Scholl, H. 2002, Icarus, 159, 328

Marzari, F., Tricarico, P., \& Scholl, H. 2003, MNRAS, 345, 1091

Mikkola, S., \& Innanen, K. 1992, AJ, 104, 1641

Mikkola, S., \& Innanen, K. 1995, Earth, Moon and Planets, 71, 195

Milani, A. 1993, CMDA, 57, 59

Milani, A. 1994, in Asteroids, Comets, Meteors, ed. A. Milani, M. Di Martino, \& A. Cellino (Kluwer), 159

Milani, A., Nobili, A., \& Knežević, Z. 1997, Icarus, 125, 13

Morais, M. H. M. 1999, A\&A, 350, 318

Morbidelli, A., Levison, H. F., Tsiganis, K., \& Gomes, R. 2005, Nature, 435, 462

Namouni, F. 1999, Icarus, 137, 293

Namouni, F., \& Murray, C. D. 2000, CMDA, 76, 131

Namouni, F., Christou, A. A., \& Murray, C. D. 1999, Physical Review Letters, 83,13

Pál, A., \& Süli, Á. 2004, in Proceedings of the British-Hungarian N+N Workshop for Young Researchers On Computer processing and use of satellite data in astronomy and astrophysics and 3rd Workshop of Young Researchers in Astronomy \& Astrophysics, ed. E. Forgács-Dajka, K. Petrovay, \& R. Erdélyi, 285

Peale, S. J. 1999, Icarus, 106, 308

Pilat-Lohinger, E., Dvorak, R., \& Tsiganis, K. 1999, CMDA, 73, 117

Rabe, E. 1967, AJ, 72, 10

Rabe, E. 1968, AJ, 73, 732

Robutel, P., Gabern, F., \& Jorba, A. 2005, CMDA, 92, 53

Sándor, Zs., \& Érdi, B. 2003, CMDA, 86, 301

Sándor, Zs., Érdi, B., \& Murray, C. D. 2003, CMDA, 84, 355

Schwarz, R., \& Gyergyovits, M. 2003, in Proceedings of the 3rd AustrianHungarian workshop on Trojans and related topics, ed. F. Freistetter, R. Dvorak, \& B. Érdi (Eötvös University Press), 71

Schwarz, R., Gyergyovits, M., \& Dvorak, R. 2004, CMDA, 90, 139

Schubart, J., \& Bien, R. 1987, A\&A, 175, 299

Shoemaker, E., Shoemaker, C., \& Wolfe, R. 1989, in Asteroids II, ed. R. Binzel, T. Gehrels, \& M. Matthews (University of Arizona Press), 487

Skokos, C., \& Dokoumetzidis, A. 2001, A\&A, 367, 729

Tsiganis, K., Dvorak, R., \& Pilat-Lohinger, E. 2000, A\&A, 354, 1091

Tsiganis, K., Varvoglis, H., \& Dvorak, R. 2005, CMDA, 92, 71 